\title{
LEVANTAMENTO DAS CONDIÇÕES DE CÁRIE E DOENÇA PERIODONTAL NA ASSOCIAÇÃO DE PORTADORES DA SÍNDROME DE DOWN EM TERESÓPOLIS-RJ
}

\author{
SURVEY OF TERMS OF CARIES AND PERIODONTAL DISEASE IN THE \\ ASSOCIATION OF THE HOLDERS OF DOWN SYNDROME IN \\ TERESOPOLIS-RJ, BRAZIL
}

\author{
Sandro Seabra Gonçalves * \\ Cláudia da Silva Emílio Canalli ** \\ Simone Guida Babinski ** \\ João Wesley Babinski **** \\ José Massao Miasato *...*
}

\section{RESUMO}

Introdução: As pesquisas na área de saúde dedicadas ao estudo de pacientes portadores de necessidades especiais vêm aumentando o interesse de muitos profissionais no atendimento desses indivíduos, que requerem atenção diferenciada. Essa afirmativa torna importante o conhecimento para o atendimento dos portadores de síndrome de Down que apresentam características próprias. Métodos: O estudo avaliou a prevalência de cárie e doença periodontal numa Associação de Portadores da Síndrome de Down no município de Teresópolis-RJ. O grupo constituiu-se de 27 indivíduos com síndrome de Down, sendo 16 do gênero feminino e 11 do masculino, com faixa etária variando de 1 a 26 anos. Para a experiência de cárie foram utilizados os índices ceo-s e CPO-S e para doença periodontal o índice IG (índice gengival). Os exames bucais foram realizados por um único avaliador nas residências dos indivíduos, os dados coletados eram anotados em uma ficha específica e cadastrados posteriormente em um banco de dados. Resultados: Num total de 2558 superfícies avaliadas, a experiência de cárie foi observada em 135 (5,27\%), sendo que em 1637 do gênero feminino observou-se ceo-s e CPO-S de 102 (6,23\%), enquanto no masculino das 921 superfícies, o ceo-s e CPO-S foi de 33 (3,58\%). Já para a doença periodontal notou-se que 59,25\% dos indivíduos apresentavam sinais clínicos de alteração no periodonto. Conclusão: Os pacientes portadores da síndrome de Down da associação em Teresópolis apresentaram baixa prevalência de cárie e alta de doença periodontal.

DESCRITORES: Cárie dentária • Doenças periodontais • Síndrome de Down.

\section{ABSTRACT}

Introduction: The research in health care dedicated to the study of patients with special needs is increasing the interest of many professionals in the care of these individuals, which require different attention. Based on so it is important knowledge to the care patients with Down syndrome which have their own characteristics. Methods: It was analyzed the prevalence of caries and periodontal disease in an association of holders of Down syndrome in the city of Teresopolis-RJ, Brazil. The group consisted of 27 individuals with Down syndrome, 16 females and 11 males, ranging in age from 1 to 26 years old. For the experience of caries were used the indices ceo-s and CPO-S and the index for periodontal disease GI (gingival index). The oral examinations were performed by a single assessor in homes of individuals, collected data were recorded in a separate sheet and subsequently registered in a specific bank. Results: In a total of 2558 areas assessed, the experience of caries was observed in 135 (5.27\%), whereas in the 1637 female there was ceo-s and CPO-S $102(6.23 \%)$, while the male of the 921 areas, the ceo-s and CPO-S was 33 (3.58\%). For periodontal disease it was noted that $59.25 \%$ of the individuals had clinical signs of change in the periodontium. Conclusion: The patients of Down syndrome in Teresópolis-RJ, Brazil had low prevalence of caries and high periodontal disease.

DESCRIPTORS: Dental caries • Periodontal diseases • Down syndrome.

\footnotetext{
* Mestrando em Odontopediatria pela Universidade da Grande Rio - Unigranrio - sandroseabra@hotmail.com

** Mestrando em Odontopediatria pela Universidade da Grande Rio - Unigranrio - ccanalli@click21.com.br

*** Professora da disciplina de pacientes especiais do Centro Universitário Serra dos Órgãos - Unifeso - sbabinski@gmail.com

**** Professor da disciplina de pacientes especiais do Centro Universitário Serra dos Órgãos - Unifeso - jbabinski@gmail.com

***** Coordenador do curso de mestrado em Odontologia/Odontopediatria da Universidade da Grande Rio Unigranrio - jmassao@gmail.com
} 
GONÇALVES SS

CANALLI CSE

BABINSKI SG

BABINSKI JW

MIASATO JM

LEVANTAMENTO

DAS CONDIÇÕES

DE CÁRIE

E DOENÇA

PERIODONTAL

NA ASSOCIAÇÃO

DE PORTADORES

DA SIINDROME

DE DOWN EM

TERESÓPOLIS - RJ

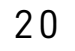

REVISTA DE ODONTOLOGIA DA UNI VERS I DADE CIDADE DE SÃO

PAULO

$2010 ; 22(1): 19-$

$24, J A N-A B R$

\section{N T RO DUÇÃ 0}

A síndrome de Down foi primeiramente descrita pelo médico inglês John Langdon Down, em 1866, baseando-se em características físicas associadas com o funcionamento orgânico. Na ocasião, Down denominou-a de "Síndrome da Idiotia Mongoliana", devido a semelhanças físicas dos indivíduos portadores da síndrome de Down às pessoas da raça mongoliana. Lanes e Vilhena-Moraes ${ }^{1} 1997$, entretanto, Schreiner afirmaram que o uso do termo era inapropriado e poderia até ofender famílias envolvidas (MacDonald e Avery ${ }^{2}$ 2001).

A síndrome de Down é uma aberração cromossômica que constitui uma das principais causas da deficiência mental de origem pré-natal. Resulta de um erro na distribuição cromossômica durante a divisão celular após a fertilização do óvulo pelo espermatozoide, que pode se apresentar de três formas (Angélico ${ }^{3}$ 2004):

- trissomia 21 (3 cromossomos 21, ao invés dos 2 habituais): o indivíduo apresenta 47 cromossomos em todas as células, com um cromossomo extra. Representa a maioria dos casos (95\%).

- mosaico: a pessoa apresenta uma mistura de células tanto normais quanto trissômicas (2\%).

- translocação: há uma fusão de 2 cromossomos, na maioria das vezes o 21 e o 15 , resultando em um total de 46 cromossomos, apesar da presença de um cromossomo 21 extra aderido a um outro par $(2 \%$ a $3 \%)$.

A síndrome de Down é considerada a anomalia mental congênita mais comum, ocorre uma vez em aproximadamente 600 a 800 nascidos com vida, sendo encontrada em $10 \%$ a $18 \%$ dos indivíduos institucionalizados por retardo mental. Tal condição independe de classe social ou raça e acomete igualmente ambos os sexos (Oliveira et al. ${ }^{4} 2001$ ).

O paciente com síndrome de Down apresenta uma série de alterações sistêmicas e bucofaciais que o cirurgião-dentista precisa conhecer para executar com êxito um atendimento com qualidade (Sasaki et al. ${ }^{5}$ 2004).

Na cavidade bucal de indivíduos com síndrome de Down observam-se algumas características peculiares e outras, presentes também em diversas patologias, como por exemplo: hipodesenvolvimento do terço médio da face, com a presença de pseudo-prognatismo, palato duro menor e ogival. Pode-se notar a presença de hipotonia lingual. Algumas anomalias dentárias também podem ser observadas, tais como: presença de hipodontia ou oligodontia, dentes conoides, microdentes, hipocalcificação do esmalte, fusão e geminação (Campioni e Guaré ${ }^{6}$ 2000). As anomalias dentárias são muito prevalentes na dentição permanente, ocorrendo com uma frequência cinco vezes maior do que na população normal (Mugayar ${ }^{7}$ 2000). Ao verificar a prevalência de cárie dentária nota-se que é relativamente baixa, porém alta para a doença periodontal (Lanes e Vilhena-Moraes ${ }^{1}$ 1997, Fiorati et al. ${ }^{8}$ 1999, Brown e Cunningan ${ }^{9}$ 1961, Mateus ${ }^{10} 1992$, Moraes et al. ${ }^{11} 2002$, Orner ${ }^{12}$ 1976). A baixa prevalência de cárie pode ser explicada pelo atraso na erupção dos dentes permanentes e pelo alto número de diastemas existentes, reduzindo consideravelmente o índice de cáries proximais (Fiorati et al. ${ }^{8}$ 1999). Na doença periodontal (Santos ${ }^{13}$ 2003), considera-se que a deficiência imunológica, provavelmente, seja o fator de maior contribuição para sua evolução, já que o organismo exibe dificuldades de combater as bactérias que estão presentes no biofilme dental.

Este trabalho tem como objetivo avaliar a prevalência de cárie e doença periodontal em indivíduos da Associação de Síndrome de Down no município de Teresópolis-RJ.

\section{MÉTODOS}

O presente trabalho foi submetido a aprovação pelo Comitê de Ética em Pesquisa do Centro Universitário Serra dos Órgãos sob o n ${ }^{\circ}$ 050/06, de acordo com a Resolução 196/96 do Conselho Nacional de Saúde e suas complementares.

A população constitui-se de 27 pacientes portadores da síndrome de Down, sendo 16 do gênero feminino e 11 do masculino, com idades variando de 1 a 26 anos. Os indivíduos são matriculados na Associação de Síndrome de Down de Teresópolis (ASSIND). Ressalta-se que eles 
não recebem atendimento odontológico regularmente, orientação de técnica de escovação e procedimentos profiláticos. Os indivíduos que apresentaram uma condição bucal insatisfatória eram selecionados para tratamento imediato.

Adotou-se o critério de estratificar a população em três grupos etários, de 1 a 9 anos, de 10 a 18 e de 19 a 26, considerando-se assim, grupos de crianças, adolescentes e adultos respectivamente. Elaborou-se uma ficha de exame direcionada para a anotação de dados obtidos. Os responsáveis foram informados da pesquisa e caso aceitassem autorizavam a participação de seus filhos, assinando o termo de consentimento livre e esclarecido.

Para a obtenção dos dados foi necessário o exame clínico e sua avaliação dada pelos índices ceo-s e CPO-S descritos originalmente por Klein e Palmer em 1937 (Gomes Pinto ${ }^{14}$, 2000). Através do Índice Gengival (IG), descrito por Löe e Silness em 1963, avaliaram-se as condições de saúde dos tecidos gengivais.

As crianças foram examinadas em suas residências pelo autor da pesquisa, sob iluminação natural, materiais e instrumentais necessários (espelho intrabucal e sonda periodontal) (Gomes Pinto ${ }^{14} 2001$ ).

Os índices ceo-s, CPO-S e Gengival fo- ram analisados de forma percentual, objetivando descrever a distribuição da experiência de cárie e doença periodontal.

A prevalência de indivíduos livres de cárie também foi levantada, através do percentual de indivíduos com ceo-s e CPO-S igual a zero.

\section{RESULTADOS}

A população estudada na faixa etária de 1 a 9 anos mostrou um maior número de indivíduos do gênero masculino. Entretanto, na faixa etária de 10 a 18 anos não foi observada a presença de nenhum paciente do gênero masculino. Nos indivíduos entre 19 a 26 anos a população feminina também foi maior.

A distribuição dos portadores de síndrome de Down estudada quanto ao número de indivíduos e de dentes decíduos e permanentes, por gênero e faixa etária, pode ser observada na Tabela 1.

Com o percentual de $6,23 \%$ das superfícies dentárias cariadas no gênero feminino e 3,58\% no masculino pode-se afirmar que $5,27 \%$ do grupo total apresentaram experiência de cárie.

O percentual de indivíduos livres de experiência de cárie não foi tão significativo, pois dos 27 indivíduos examinados, 10 não apresentavam experiência de cá-

Tabela 1 - Número de indivíduos, dentes decíduos e permanentes por gênero e faixa etária

\begin{tabular}{ccccccc} 
GÊNERO & \multicolumn{3}{c}{ FEMININO } & \multicolumn{3}{c}{ MASCULINO } \\
IDADE & $\begin{array}{c}N^{\circ} \\
\text { Indivíduos }\end{array}$ & $\begin{array}{c}N^{\circ} \text { Dentes } \\
\text { Decíduos }\end{array}$ & $\begin{array}{c}N^{\circ} \text { Dentes } \\
\text { Permanentes }\end{array}$ & $\begin{array}{c}N^{\circ} \\
\text { Indivíduos }\end{array}$ & $\begin{array}{c}N^{\circ} \text { Dentes } \\
\text { Decíduos }\end{array}$ & $\begin{array}{c}N^{\circ} \text { Dentes } \\
\text { Permanentes }\end{array}$ \\
$10-18$ & 5 & 41 & 39 & 8 & 115 & 10 \\
$19-26$ & 5 & 8 & 136 & - & - & - \\
Total & 16 & 43 & 125 & 3 & 9 & 71 \\
\hline \hline
\end{tabular}

Tabela 2 - Prevalência de cárie de acordo com ceo-s + CPO-S por faixa etária em ambos os gêneros

\begin{tabular}{cccccccc} 
IDADE & \multicolumn{2}{c}{ ceo-s + CPO-S } & \multicolumn{2}{c}{ TS } & \multicolumn{2}{c}{ \% ceo-s + CPO-S } \\
& F & M & F & M & F & M \\
$01-09$ & 23 & 16 & 357 & 556 & 6,44 & 2,87 \\
$10-18$ & 32 & - & 692 & - & 4,62 & - \\
$19-26$ & 47 & 17 & 588 & 365 & 7,99 & 4,65 \\
Total & 102 & 33 & 1637 & 921 & 6,23 & 3,58 \\
\hline \hline
\end{tabular}

ceo-s + CPO-S = total de superfícies com experiência de cárie

$\mathrm{TS}=$ total de superfícies

\% ceo-s + CPO-S = percentual de superfícies com experiência de cárie

$\mathrm{F}=$ feminino

$M=$ masculino

GONÇALVES SS

CANALLI CSE

BABINSKI SG

BABINSKI JW

MIASATO JM

LeVANTAMENTO

DAS CONDIÇÕES

DE CÁRIE

E DOENÇA

PERIODONTAL

NA ASSOCIAÇÃO

DE PORTADORES

DA SINDROME

DE DOWN EM

TERESÓPOLIS-RJ 
GONÇALVES SS

CANALLI CSE

BABINSKI SG

BABINSKI JW

MIASATO JM

LEVANTAMENTO

DAS CONDIÇÕES

DE CÁRIE

E DOENÇA

PERIODONTAL

NA ASSOCIAÇÃO

DE PORTADORES

DA SIINDROME

DE DOWN EM

TERESÓPOLIS-RJ

REVISTA DE

ODONTOLOGIA DA

UNIVERSIDADE

CIDADE DE SÃO

PAULO

$2010 ; 22(1): 19-$

24, JAN-ABR

Tabela 3 - Número e percentual de indivíduos livres da experiência de cárie por gênero e faixa etária

\begin{tabular}{|c|c|c|c|c|c|c|}
\hline SEXO & & FEMININO & & & MASCULINO & \\
\hline IDADE & $\begin{array}{c}\mathrm{N}^{\mathrm{o}} \\
\text { Indivíduos }\end{array}$ & $\begin{array}{l}\text { No Indivíduos } \\
\text { Livres de Cárie }\end{array}$ & $\begin{array}{c}\text { \% Indivíduos } \\
\text { Livres de } \\
\text { Cárie }\end{array}$ & $\begin{array}{c}\mathrm{N}^{\mathrm{o}} \\
\text { Indivíduos }\end{array}$ & $\begin{array}{l}\text { No Indivíduos } \\
\text { Livres de Cárie }\end{array}$ & $\begin{array}{c}\text { \% Indivíduos } \\
\text { Livres de } \\
\text { Cárie }\end{array}$ \\
\hline $01-09$ & 5 & 2 & 40,00 & 8 & 4 & 50,00 \\
\hline $10-18$ & 6 & 2 & 33,33 & - & - & - \\
\hline $19-26$ & 5 & 1 & 20,00 & 3 & 1 & 33,33 \\
\hline Total & 16 & 5 & 31,25 & 11 & 5 & 45,45 \\
\hline
\end{tabular}

Tabela 4 - Número e percentual de indivíduos com doença periodontal por gênero, faixa etária e níveis

\begin{tabular}{|c|c|c|c|c|c|c|c|c|}
\hline \multirow{3}{*}{ IDADE } & \multicolumn{4}{|c|}{ FEMININO } & \multicolumn{4}{|c|}{ MASCULINO } \\
\hline & \multicolumn{4}{|c|}{ NÍVEIS (\%) } & \multicolumn{4}{|c|}{ NÍVEIS (\%) } \\
\hline & 0 & 1 & 2 & 3 & 0 & 1 & 2 & 3 \\
\hline $01-09$ & 80 & 20 & - & - & 62,5 & 37,5 & - & - \\
\hline $10-18$ & 16,6 & 83,3 & - & - & - & - & - & - \\
\hline $19-26$ & 20 & 20 & 60 & - & - & 100 & - & - \\
\hline
\end{tabular}

rie, podendo-se afirmar que 37,03\% da população não apresentaram sinais clínicos presentes ou anteriores de cárie.

Através do índice IG observou-se a condição periodontal desses pacientes, também com divisão de gênero e faixa etária; para tal, subdividiu-se em níveis. Nota-se que a maioria da população classificada no nível 1 não observou nenhuma estabelecida para o nível 3. No gênero masculino não houve classificação do nível 2.

Além disso, também foi avaliado o percentual total dos níveis por sexo, observando-se que no feminino $37,5 \%$ eram classificados no nível 0 , com $43,7 \%$ no nível 1 e 18,7\% no nível 2. Já no masculino $45,4 \%$ no nível 0 e $54,5 \%$ no nível 1. No total, nota-se que dos 27 pacientes estudados, apenas 11 (40,74\%) não apresentaram sinais clínicos de alteração no tecido periodontal contra 16 (59,25\%).

\section{I SCUSSÃO}

A evolução de pesquisas na área de saúde dedicadas ao estudo de pacientes portadores de necessidades especiais vem aumentando o interesse de muitos profissionais no atendimento desses indivíduos, que requerem atenção diferenciada. Entretanto, essa atenção não é dada por todos e muitos sentem-se despreparados em atender tais necessidades.

Os portadores da síndrome de Down apresentam características próprias, principalmente em relação às condições da cavidade bucal, destacando-se a prevalência da cárie e da doença periodontal, o que envolve mais um fator de saúde para esses indivíduos. Na literatura foram encontradas maneiras diversificadas para a metodologia aplicada, levando-se em consideração os índices, ambiente e faixa etária. Entretanto, os autores são unânimes em afirmar que existe uma baixa prevalência de cárie e alta de doença periodontal nos portadores de síndrome de Down.

Além disso, o atraso na erupção dos dentes permanentes e a presença de diastemas são fatores que contribuíram para a redução do percentual de cárie.

No presente estudo, analisando-se a experiência de cárie em superfícies de dentes permanentes e decíduos (ceo-s + CPO-S), verificou-se um percentual de 6,23 no sexo feminino e 3,58 no sexo masculino, com média de 5,27 no grupo total.

No trabalho descrito por Brown e Cunningham ${ }^{9}$ (1961) foram verificados 80 pacientes com síndrome de Down, na faixa etária de 1 a 30 anos, e avaliado apenas o número de dentições livres de cárie. Em seus resultados, os pacientes mostraram uma considerável ausência de cárie, e foi notado 44\%, um valor aproximado nesta pesquisa $(37 \%)$, sendo valores aproximados em épocas diferentes e inoportunas, 
mas os resultados são semelhantes aos de Matheus $^{10}$ (1992) que encontrou $36 \%$ e diferentes dos de Fiorati et al. ${ }^{8}$ (1999) com $24 \%$.

Matheus ${ }^{10}$ (1992) pesquisou a experiência de cárie e doença periodontal em 100 portadores de síndrome de Down com idades variando de 3 a 14 anos. O índice utilizado foi o mesmo deste trabalho, permitindo assim uma comparação viável. No sexo feminino notou-se que $6,65 \%$ apresentaram sinais clínicos de experiência de cárie e 8,97\% no sexo masculino; nesses valores foram considerados os dentes decíduos e permanentes. Apesar de faixa etária diferente, foi semelhante ao resultado deste trabalho na população feminina $(6,23 \%)$, porém na população masculina em apenas 3,58\% essa diferença se deu, pelo fato de a presente pesquisa não ter indivíduos do gênero masculino na faixa etária de 10 a 18 anos, além de apresentar uma quantidade menor. Ressalta-se que nessa referência foram avaliadas as condições de cárie pela superfície dentária.

Fiorati et al. ${ }^{8}$ (1999) em seus estudos avaliaram a prevalência de cárie e doença periodontal em portadores de síndrome de Down. Foram examinados 25 pacientes, com faixa etária de 6 e 36 anos, sendo 10 pacientes do sexo masculino e 15 do sexo feminino. O autor preferiu utilizar o índice CPO-D, o que faz esperar assim um percentual diferente, fazendo com que 32\% dos pacientes apresentassem alta incidência de cárie, $40 \%$ média e $4 \%$ baixa.

Moraes et al. ${ }^{11}$ (2002) avaliaram a prevalência de cárie em 38 portadores de síndrome de Down, utilizando o índice CPO-D, com indivíduos de 3 a 28 anos de idade, sendo 21 do gênero masculino e 17 do feminino. Através da amostra estuda$\mathrm{da}$, os autores verificaram os índices ceo-d com 1,15\%, CPO-D de 2,68 e de ceo-d
+ CPO-D de 3,84\%. Apesar da diferença dos índices também foi comprovada uma baixa prevalência de cárie, diferentemente do anterior. Existe uma dificuldade para comparar os resultados, pois nesta pesquisa avaliou-se superfícies dentárias e não apenas dentes. Os trabalhos de experiência de cárie em portadores de síndrome de Down encontrados na literatura não permitiram uma melhor comparação de resultados, mostrando, assim, a necessidade de serem realizadas novas pesquisas na área com metodologia semelhante.

Para a condição periodontal, observase um decréscimo no desenvolvimento da doença. Dos 27 pacientes estudados, nota-se que em 16 houve alguma alteração do tecido gengival, o que dá um percentual de $59,25 \%$, sendo menor se comparado com os trabalhos de Brown, Cunningham ${ }^{9}$ (1961) - 90\%, Orner ${ }^{12}$ (1976) - 89\% e Fiorati et al. ${ }^{8}$ (1999) - 72\%. Entretanto, tal fato faz pensar que, independente das metodologias diversificadas, do interesse e o grau de informação dos responsáveis, o controle da higiene vem aumentando com o tempo. Contudo, o percentual de doença periodontal ainda é alto em idades precoces. Para tal, são necessárias estratégias de promoção de saúde com esses indivíduos e seus responsáveis, objetivando uma meIhor condição de saúde bucal.

A literatura carece de maiores estudos na avaliação da prevalência de cárie e doença periodontal no portador da síndrome de Down, tendo em vista o reduzido número de trabalhos publicados.

\section{CONCLUSÃO}

Os portadores da síndrome de Down da associação em Teresópolis-RJ apresentaram baixa prevalência de cárie e alta de doença periodontal.
GONÇALVES SS CANALLI CSE BABINSKI SG BABINSKI JW MIASATO JM

LEVANTAMENTO DAS CONDIÇÕES DE CÁRIE

E DOENÇA PERIODONTAL NA ASSOCIAÇÃO DE PORTADORES DA SIINDROME DE DOWN EM TERESóPOLIS-RJ
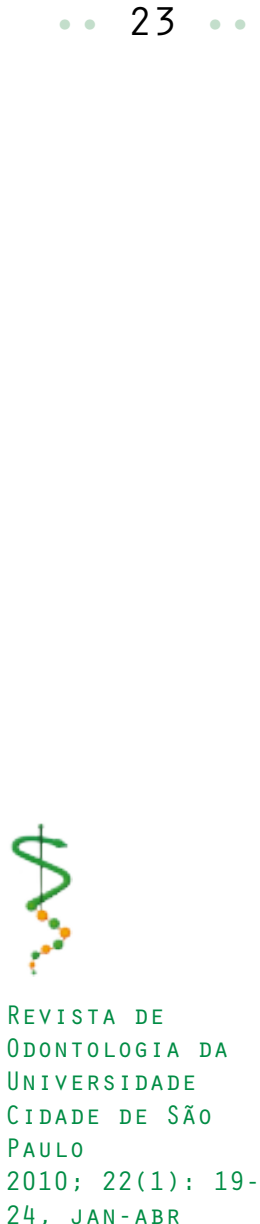

24 , JAN-ABR 


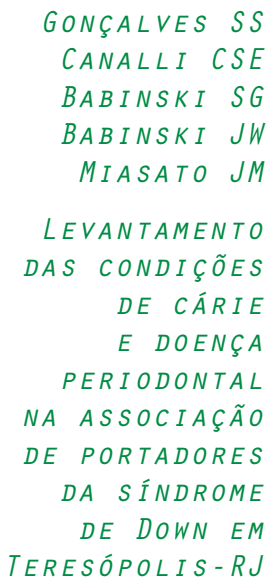

\section{REFERÊNCIAS}

1. Lannes C, Vilhena-Moraes SA. Pacientes especiais. In: Guedes Pinto AC. Odontopediatria, 6a ed. São Paulo: Santos; 1997, p. 875-904.

2. McDonald RE, Avery DR. Erupção dos dentes: fatores locais, sistêmicos e congênitos que influenciam o processo. In: Odontopediatria, $7^{\text {a }}$ ed. Rio de Janeiro: Guanabara Koogan; 2001, p.129-150.

3. Angélico AP. Estudo descritivo do repertório de habilidades sociais de adolescentes com síndrome de Down [Dissertação]. São Paulo: Universidade de São Paulo, 2004.

4. Oliveira ACB, Jorde ML, Paiva SM. Aspectos relevantes à abordagem odontológica da criança com síndrome de Down. Rev. CROMG. 2001; 7(1): 36-42.

5. Sasaki Y. et al. Periodontal management of an adolescent with Down's syndrome: a case report. Int J Paediatr Dent 2004 mar; 14(2): 127-30.

6. Ciamponi AL, Guaré RO. A criança com Trissomia do cromossomo 21 (Síndrome de Down). In: Odontopediatria: resoluções clínicas. Curitiba: Maio; 2000, p. 301312.

7. Mugayar LRF. Fundamentos da genética clínica e principais enfoques odontológicos. In: Pacientes portadores de necessidades especiais: manual de Odontologia e saúde oral. Rio de Janeiro: Pancast, 2000, p. 47-110.

8. Fiorati SM, Spósito RA, Borsatto MC. Prevalência de cárie dentária e doença periodontal em pacientes com síndrome de Down. Odontol 2000. 1999 jul-dez; 3(2): 58-62.

9. Brown RH, Cunningham WM. Some dental manifestations of mongolism. Oral Surg Med Oral Pathol 1961 Jun; 14(6): 664-76

10. Matheus WD. Levantamento das condições de cárie e doença periodontal do paciente portador de síndrome de Down institucionalizado na APAE-RJ. [Dissertação]. Rio de Janeiro: Universidade Federal do Rio de Janeiro; 1992.

11. Moraes MEL. et al. Prevalência de cárie pelo índice CPO-D em portadores de síndrome de Down. PGR: Pós-Grad Rev Odontol 2002 maio-ago; 5(2): 64-73.

12. Orner G. Periodontal disease among children with Down's syndrome and their siblings. J Dent Res 1976 Sep-Oct; 55(5): 778-782.

13. Santos P. Pacientes com síndrome de Down apresentam doença periodontal precoce. Rev. Sobrape. 2003 Abr-Jun; 81: 53-57.

14. Gomes Pinto V. Identificação de problemas. In: Saúde bucal coletiva, 4 a ed. São Paulo: Santos; 2000, p. 139-222.

Recebido em: 14/09/2009

Aceito em: 11/01/2010 